\title{
Rising and Sinking in Resonance: Mass Distribution Critically Affects Buoyancy-Driven Spheres via Rotational Dynamics
}

\author{
Jelle B. Will $\odot^{*}$ and Dominik Krug $\odot^{\dagger}$ \\ Physics of Fluids Group and Max Planck Center Twente, J. M. Burgers Centre for Fluid Dynamics, \\ University of Twente, P.O. Box 217, 7500 AE Enschede, Netherlands
}

(Received 7 December 2020; accepted 7 April 2021; published 30 April 2021)

\begin{abstract}
We present experimental results for spherical particles rising and settling in a still fluid. Imposing a wellcontrolled center of mass offset enables us to vary the rotational dynamics selectively by introducing an intrinsic rotational timescale to the problem. Results are highly sensitive even to small degrees of offset, rendering this a practically relevant parameter by itself. We further find that, for a certain ratio of the rotational to a vortex shedding timescale (capturing a Froude-type similarity), a resonance phenomenon sets in. Even though this is a rotational effect in origin, it also strongly affects translational oscillation frequency and amplitude, and most importantly, the drag coefficient. This observation equally applies to both heavy and light spheres, albeit with slightly different characteristics for which we offer an explanation. Our findings highlight the need to consider rotational parameters when trying to understand and classify path properties of rising and settling spheres.
\end{abstract}

DOI: 10.1103/PhysRevLett.126.174502

A single particle settling or rising in a still fluid is one of the most intuitive and conceptually simple problems in fluid mechanics. However, the complexity arising from coupling between the motion of the body and the surrounding flow is intricate, and the resulting complex trajectories [1-6] have fascinated researchers, including Da Vinci [7] and Newton [8], for centuries. Moreover, single particle dynamics often persist in particle-laden flows [9] and affect global properties of a system such as sedimentation rate, transport of heat or nutrients in a fluid [10], or mixing for chemical reactors [11,12]. Beside the scientific appeal, a fundamental understanding of the behavior of individual particles is, therefore, of primary importance in understanding larger systems in nature and industrial applications.

Despite long-standing efforts, the understanding even for the most basic geometry of a sphere is still incomplete to date $[13,14]$. The traditional notion is that the two-way coupled dynamics for this case depend on two dimensionless parameters only: the particle-to-fluid mass density ratio $\Gamma \equiv \rho_{p} / \rho_{f}$, and the particle Galileo number Ga $\equiv U_{b} D / \nu$ $[15,16]$. Here, $D$ is the particle diameter, $\nu$ the kinematic viscosity of the fluid, and $U_{b}=\sqrt{|1-\Gamma| g D}$ is the buoyancy velocity with $g$ denoting the acceleration due to gravity. In relating buoyancy and viscous forces, $\mathrm{Ga}$ is similar to the Reynolds number $\operatorname{Re} \equiv\left\langle u_{z}\right\rangle D / \nu$, where $\left\langle u_{z}\right\rangle$ is the mean vertical velocity (with $\langle\cdot\rangle$ denoting a time and ensemble average) which is not known a priori, however.

A significant amount of work was aimed at classifying the motion of spheres and differences in their wake structures as a function of $\Gamma$ and Ga [13,16-20]. However, there still exists substantial disagreement even on fundamental aspects. For example, it remains open why there are conflicting results for the parameter range for which strong path oscillations are observed [15,17-26]. The lack of a universal description alludes to the possibility that additional-yet largely unexplored-parameters may play a role. In fact, recently, the importance of rotational dynamics for spheres and 2D cylinders has been highlighted [14,27,28], showing that the moment of inertia (MOI, governed by the internal mass distribution) can affect the vortex shedding mode, the frequency and amplitude of oscillation, and the vertical velocity. The key physical mechanism behind this rotational-translational coupling is the Magnus lift force, which, in a still fluid, is given by $\boldsymbol{F}_{m} \sim \boldsymbol{\omega} \times \boldsymbol{u}$ [29], with $\boldsymbol{\omega}$ and $\boldsymbol{u}$ denoting particle angular and linear velocity vectors, respectively. It has been suggested that the dependence on particle MOI can be one of the factors contributing to the spread in particle drag coefficient as well as causing differences in oscillation amplitude [14], but conclusive evidence, in particular for spheres, is missing.

In this Letter, we systematically explore the effect of rotational dynamics on rising and settling spheres. To this end, we modify the rotational properties of the spherical particles in a controlled manner by introducing a center of mass (c.m.) offset $\gamma \equiv 2 l / D$, where $l$ is the distance along the unit vector $\boldsymbol{p}$ pointing from the c.m. to the geometrical center [see Fig. 1(a)]. Clearly, such an offset can also be expected to occur in a host of practical applications, where particle properties are rarely ever uniform. This concerns, for example, the falling of dandelion seeds [30] and snowflakes [31-35], the sedimentation behavior of sand 


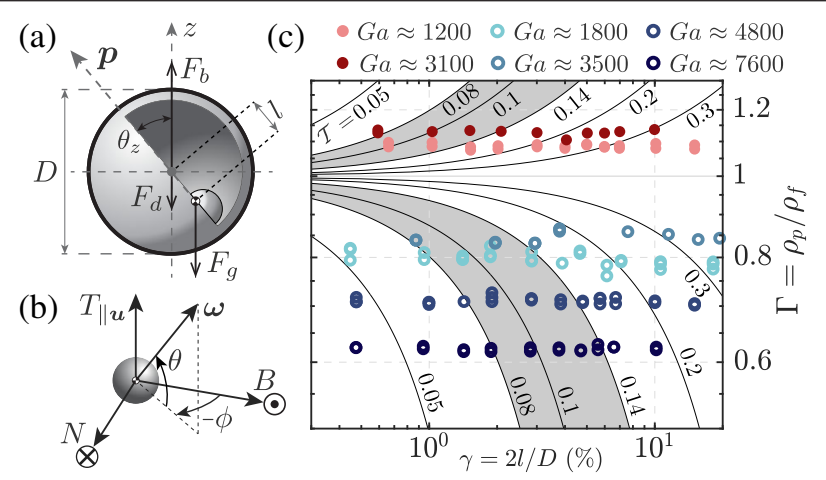

FIG. 1. (a) Schematic of a sphere with c.m. offset. (b) The particle Frenet-Serret (TNB) coordinate system, with unit vectors $\boldsymbol{T}$ (parallel to $\boldsymbol{u}$ ), $\boldsymbol{N}$ (pointing in the direction of curvature of the path), and $\boldsymbol{B}$ (defined such that $\boldsymbol{N}=\boldsymbol{B} \times \boldsymbol{T}$ ). The angles $\phi$ (azimuth) and $\theta$ (elevation) uniquely define a vector in this space. (c) Explored parameter space. Grey shading indicates the resonance regime and $\mathcal{T}$ isocontours correspond to $I^{*}=1$.

grains and stones [36,37], chemical and biological reactors with (inverse) fluidized beds [38], as well as the transport of microplastic in the oceans [39]. Moreover, the practical relevance is rooted in the fact that we find that even small values of $\gamma$ can affect the kinematics and dynamics of spherical particles significantly. Despite their apparent relevance, c.m. offsets are often listed more generally as potential sources of experimental uncertainty (e.g., [5]), but few studies have considered $\gamma$ explicitly. To our knowledge, the relevance of this parameter was first noted by Jenny et al. [17] who report that the trajectory of a settling sphere with $\mathrm{Ga}=180$ was destabilized when introducing an offset of $\gamma \approx 5 \%$ (originating from air bubbles occasionally trapped inside their particles). More recently, it was shown that lateral motion of spheres in a linear shear flow was reduced by presence of a strong offset [40]. While both of these studies clearly underline the relevance of $\gamma$ as a parameter, the accounts remain anecdotal and a complete understanding based on systematic variation is lacking still. For completeness, it should be mentioned that the role of mass asymmetry has also been examined in the context of cylindrical or fiberlike particles [41-43]. However, due to the anisotropic geometry, the dynamics in these instances are completely different from the spherical case considered here.

We start our analysis from the classical Kelvin-Kirchhoff equations [44], which, for a suspended sphere, are given by

$$
\begin{gathered}
\left(1+\frac{1}{2 \Gamma}\right)\left(\frac{d \boldsymbol{u}}{d t}+\boldsymbol{\omega} \times \boldsymbol{u}\right)=\frac{\boldsymbol{F}_{f}}{m_{p}}+\frac{(1-\Gamma) g}{\Gamma} \boldsymbol{e}_{z}, \\
\frac{1}{10} I^{*} \frac{d \boldsymbol{\omega}}{d t}=\frac{\boldsymbol{T}_{f}}{m_{p} D^{2}}-\frac{\gamma}{2 D}\left(\boldsymbol{a}_{c}+g \boldsymbol{e}_{z}\right) \times \boldsymbol{p} .
\end{gathered}
$$

Here, $\boldsymbol{F}_{f}$ and $\boldsymbol{T}_{f}$ are the fluid force and torque applied to the body, respectively, and $\boldsymbol{e}_{z}$ is the vertical unit vector.
Further, we define the dimensionless MOI $I^{*} \equiv I_{p} / I_{\Gamma}$ as the ratio of the particle MOI over the MOI of a sphere with a uniform density distribution $I_{\Gamma}=1 / 10 m_{p} D^{2}$, where $m_{p}$ is the particle mass. Note that the linear momentum balance [Eq. (1)] remains unaffected by the choice of $\gamma$. Equation (2) represents the angular momentum balance around the center of the sphere, in which the effect of the c.m. offset appears in the form of the cross product on the right-hand side. Apart from $\gamma$, the magnitude of this term also depends on the included angle $\theta_{z}$ between $\boldsymbol{p}$ and $\boldsymbol{e}_{z}$ [see Fig. 1(a)], and on $\boldsymbol{a}_{c}$, the acceleration of the center of mass.

For spheres, the geometric center and the center of pressure coincide. Therefore, the forcing term $\boldsymbol{T}_{f}$ in Eq. (2) is solely due to skin friction, which, for $\mathrm{Re} \gtrsim 275$ [45], provides an approximately periodic driving associated with the vortex shedding in the wake of the body [46]. Neglecting the additional dependence on $\boldsymbol{a}_{c}$, the offset term acts as a restoring torque. Thus, Eq. (2) is similar to a periodically forced pendulum with a natural frequency $f_{p}=\sqrt{5 \gamma g / D I^{*}} / 2 \pi$ and the corresponding timescale $\tau_{p}=f_{p}^{-1}$. The driving, due to vortex shedding, is characterized by $\tau_{v} \sim D / U_{b}$, and on this basis, we define the ratio

$$
\mathcal{T}=\frac{\tau_{v}}{\tau_{p}}=\frac{1}{2 \pi} \sqrt{\frac{5 \gamma}{|1-\Gamma| I^{*}}} .
$$

Note that $\mathcal{T}$ is entirely determined by particle properties. In relating translational $\left(U_{b}\right)$ and dissipative $\left(D / \tau_{p}\right)$ velocities, $\mathcal{T}$ corresponds to the inverse of the Froude number defined in [47] for falling strips. However, the definition in Eq. (3) is preferred, here, as it avoids a singularity at $\gamma=0$.

To test the effect of variations in $\mathcal{T}$, laboratory experiments were performed for rising (blue) and settling spheres (red symbols) in a still fluid with systematic variations in $\mathrm{Ga}, \gamma$, and $\Gamma$. An overview over the explored parameter range is shown in Fig. 1(c). Particles, $D=12-25 \mathrm{~mm}$, were released to settle or rise in a large vertical water tank. After an initial transient $(>20 D)$, the position and orientation of the spheres were tracked over a distance of $\approx 30-80 D$ using optical methods $[48,49]$. Details of the setup and the postprocessing of the data are provided in the Supplemental Material [50] that also includes movies of rendered trajectories at $G a=1800$.

The profound effect variations in $\gamma$ have on particle kinematics is exemplified in Fig. 2(a), where horizontal projections ( $X Y$ plane) of drift corrected trajectories for the $\mathrm{Ga} \approx 1800$ (rising) case are shown. From these plots, it is obvious that the oscillation amplitude varies significantly with $\gamma$ and even vanishes for the most extreme offset. Simultaneously, the shape of the oscillations also transitions from mostly planar to circular and then back to a more planar motion with additional precession as $\gamma$ is increased. A similar behavior is observed across all $\mathrm{Ga}$ and $\Gamma$ for rising particles. For $\Gamma>1$, we observed a similar increase in 

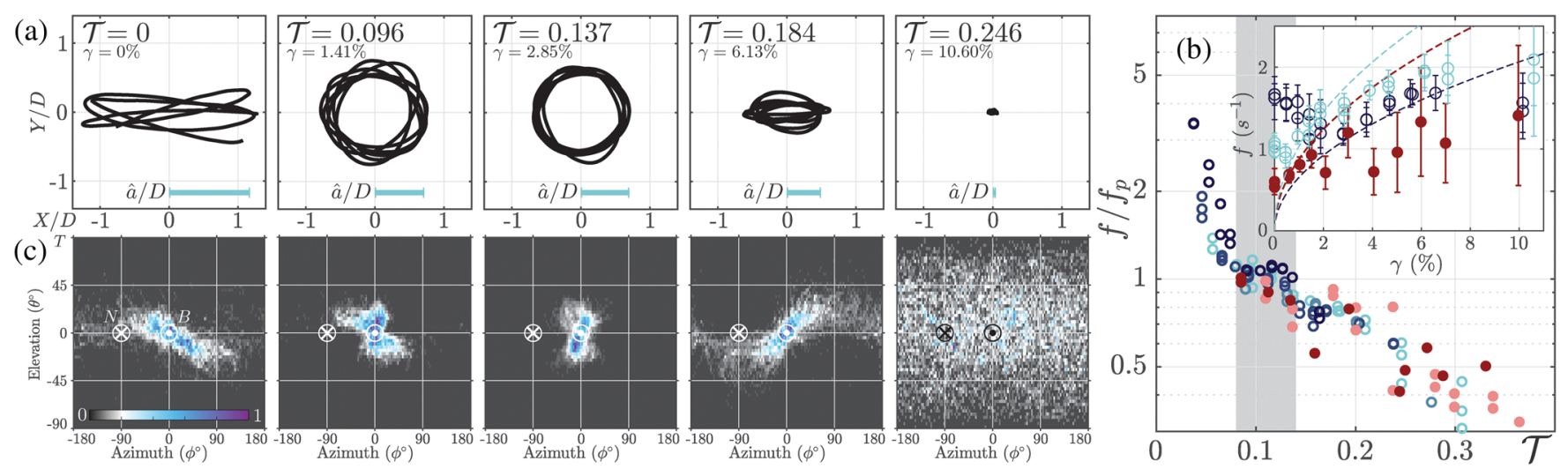

FIG. 2. (a) Characteristic trajectories of rising particles $(\mathrm{Ga} \approx 1800$ and $\Gamma \approx 0.80)$ as seen from the top for different values of $\mathcal{T}$. The length of the horizontal blue lines represent the corresponding amplitudes $\hat{a} / D$. (b) Inset: $f$ (symbols) and $f_{p}$ (dashed lines) vs $\gamma$ for three different Ga values. Main figure: ratio $f / f_{p}$ vs $\mathcal{T}$ for the entire dataset. (c) Normalized histograms of the orientation of $\boldsymbol{\omega}$ in the TNB coordinates for the cases corresponding to (a). The histograms contain data of all particles with nominally the same parameters.

amplitude but not the associated helical and precessing trajectories. Unlike reported at lower Ga [13], we did not encounter significant horizontal drift here.

As a first quantitative measure, we extract the frequency $f$ of the horizontal path oscillations. Sample results for three cases in the inset of Fig. 2(b) reveal that $f$ varies significantly with $\gamma$ with a remarkable sensitivity even at small offsets. All cases display a similar pattern relative to their respective pendulum frequency $f_{p}(\gamma)$ (dashed lines): At small $\gamma, f$ exceeds $f_{p}$, but the two quickly converge as the offset is increased resulting in a resonance $\left(f \approx f_{p}\right)$ between the path oscillations (and hence, the vortex shedding) and the rotational dynamics of the particle. For offsets greater than those at resonance, $f_{p}$ quickly outgrows the shedding frequency, and path oscillations damp out (resulting in large variations in $f$ in this regime). Resonance occurs at different values of $\gamma$ for different particles. However, all data collapse when plotting $f / f_{p}$ against $\mathcal{T}$ as is done in the main panel Fig. 2(b). This confirms that $\mathcal{T}$ is, indeed, the relevant parameter governing the behavior of particles with c.m. offset, and we identify the resonance range as $0.08 \lesssim \mathcal{T} \lesssim 0.14$, where $f / f_{p} \approx 1 \pm 0.2$ (marked by grey shading in all figures). A similar lock-in phenomenon of the wake to object oscillations was observed earlier for forced translational oscillations of beams in a cross flow [51,52]. A key difference and a remarkable feature of the present results is, however, that, here, vortex shedding dynamics are governed by a parameter that is intrinsically rotational.

The resonance behavior revealed for the frequencies also has a direct imprint on other parameters, such as the normalized oscillation amplitude $\hat{a} / D$ shown in Fig. 3(a) for both heavy and light particles. At $\mathcal{T}=0$, scatter in $\hat{a} / D$ is considerable owing to variation in $\mathrm{Ga}, I^{*}$, and $\Gamma$. However, these differences vanish and the variation of $\hat{a} / D$ as a function of $\mathcal{T}$ becomes remarkably similar across all cases tested, rendering this the dominant parameter once a small but finite offset $(\gamma>0)$ is introduced. Amplitudes are largest in the resonance band with a peak of $\hat{a} / D \approx 1$ located at $\mathcal{T} \approx 0.09$ for both rising and settling particles. Consistent with the observation in Fig. 2(a), path oscillations vanish at large $\mathcal{T}$ in all cases, and it appears that the decrease in $\hat{a} / D$ beyond resonance is steeper for larger values of $\Gamma$. While the resonant behavior in terms of $f / f_{p}$ and $\hat{a} / D$ is very similar for heavy and light particles, remarkably, the same is not true for the drag coefficient $C_{d}=4 D|1-\Gamma| g / 3\left\langle v_{z}\right\rangle_{t}^{2}$ shown in Fig. 3(b). For rising spheres, there is almost a factor of 2 increase in $C_{d}$ in the resonance regime as compared to the $\mathcal{T}=0$ case. In contrast, the $C_{d}$ results appear virtually insensitive to any changes in $\mathcal{T}$ for settling spheres.

A clue pointing to the cause of this surprising behavior is given by the results for the rotational amplitude $\hat{\theta}_{z}$ in Fig. 3(c). The resonance peak for $\hat{\theta}_{z}$ is prominent at low $\Gamma$ reaching values even beyond $90^{\circ}$, but remains weak for $\Gamma>1$. In all cases, the rotational amplitude vanishes for higher $\mathcal{T}$, for which $f<f_{p}$. Indeed, the scaling $\hat{\theta}_{z} \sim \mathcal{T}^{-2}$, which follows from a quasistatic assumption using $T_{f} \sim$ $\rho_{f} D^{3} U^{2}[53,54]$, appears to capture the decay of $\hat{\theta}_{z}$ with increasing $\mathcal{T}$ well in this regime. Such a simple argument fails, however, to reproduce the prefactor properly for which the suggested $\left(\Gamma I^{*}\right)^{-1}$ dependence is weaker than the actual variation in the data. Dynamically, the rotation rate is more relevant than $\hat{\theta}_{z}$, and it further provides a more robust measure, even at $\gamma=0$. Therefore, we additionally consider the mean rotation rate $\langle\omega\rangle$ in Fig. 3(d) and observe a good agreement between the trend of this quantity and that of $C_{d}$ as a function of $\mathcal{T}$. This indicates that, instead of the path oscillation amplitude (which features a resonance peak even for $\Gamma>1$ ), the particle drag correlates better with the rotational energy of the spheres. 

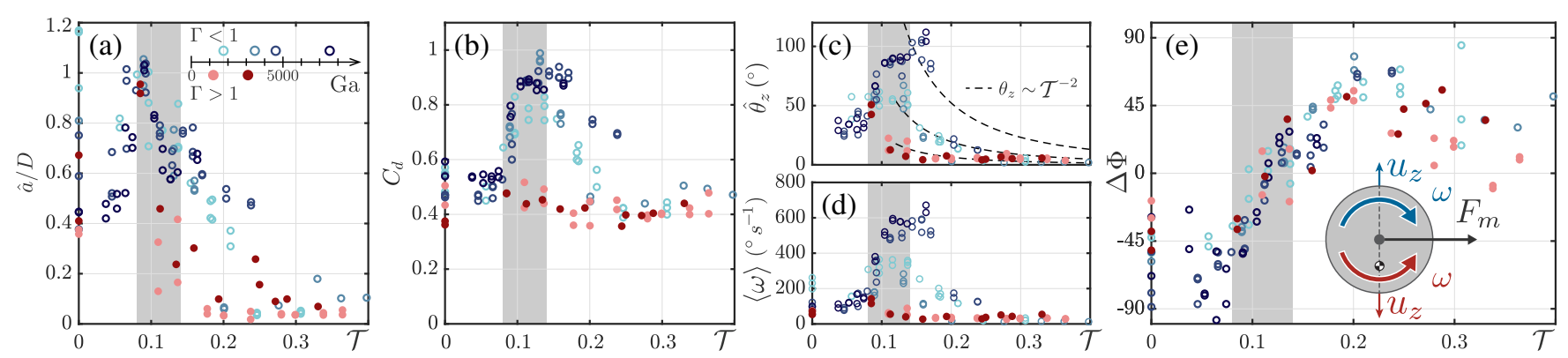

FIG. 3. Dependence on $\mathcal{T}$ for (a) amplitude of the path oscillations $\hat{a} / D$, (b) particle vertical drag coefficient $C_{d}$, (c) particle rotational amplitude $\hat{\theta}_{z}$, (d) time averaged angular velocity $\langle\omega\rangle$, (e) phase angle $\Delta \Phi$ between horizontal particle acceleration and Magnus lift force. All data points represent averages over multiple experiments with the same particle.

In evaluating the nature of the rotational-translational coupling, it is useful to consider the Lagrangian FrenetSerret coordinate system [T, $\boldsymbol{N}, \boldsymbol{B}$, see Fig. 1(b)], which is defined with respect to the path of the sphere $[14,29,55]$. In Fig. 2(c), we have included histograms of the orientation of $\boldsymbol{\omega}$ in the TNB coordinate frame corresponding to the sample trajectories displayed in Fig. 2(a). Especially for the resonance cases $(\mathcal{T}=0.096$ and $\mathcal{T}=0.137), \boldsymbol{\omega}$ is found to align strongly with $\boldsymbol{B}$. This implies that the normal acceleration (along $N$ ) is consistent with the direction of the Magnus lift force in this state, since $\boldsymbol{F}_{m} \sim \boldsymbol{\omega} \times \boldsymbol{u}$. In addition to the fact that no significant path oscillations are observed in the absence of particle rotation at high $\mathcal{T}$ (Fig. 3), this underlines the crucial role rotational dynamics play for the path oscillations. The alignment between $\boldsymbol{\omega}$ and $\boldsymbol{B}$ in the resonance range is slightly less pronounced at $\Gamma>1$ (see Supplemental Material [50]) but remains a robust feature for all cases considered here. While light particles at $\mathcal{T}$ outside resonance display distinct alignments away from $\boldsymbol{B}$, this is not observed at $\Gamma>1$, as rotational amplitude quickly vanishes in those cases.

With the relevance of the driving via the Magnus force established, it is then possible to analyze the phase relation between a forcing parameter and a system response. We do so by evaluating the phase angle $\Delta \Phi$ between the projections of the acceleration $\boldsymbol{a}$ and of the Magnus lift force $\boldsymbol{F}_{m}$ along an arbitrary horizontal direction. By definition, particle acceleration lags behind Magnus lift forcing for $\Delta \Phi<0$ and vice versa for $\Delta \Phi>0$. The results for $\Delta \Phi$ in Fig. 3(e) display a collapse as a function of $\mathcal{T}$ with a zero crossing (at $\mathcal{T} \approx$ $0.12 \pm 0.01$ ) within the resonance band. The latter is in line with the findings in Fig. 2(c) and implies an enhancement of path oscillations through $\boldsymbol{F}_{m}$. Therefore, a key feature of the resonance is that rotational-translational coupling is coherent with other forcing (e.g., through pressure forces induced by vortex shedding), while the two are less correlated, otherwise. Interestingly, $\Delta \Phi \approx 0^{\circ}$ occurs at $\mathcal{T} \approx 0.12$, at which rotations are strongest, whereas the phase lag is nonzero at the peak in $\hat{a} / D\left(\Delta \Phi \approx-45^{\circ}\right.$ at $\left.\mathcal{T} \approx 0.09\right)$.

The question remains, why the settling spheres have such a pronounced deficit in rotational dynamics compared to rising ones. An explanation for this is related to the difference in alignment between the direction of offset $\boldsymbol{p}$ (always pointing up) and the mean direction of motion, that switches between rising and settling particles. Therefore, a Magnus lift force in the same direction is associated with rotations in opposite directions between the two cases, as the inset in Fig. 3(e) shows. This is relevant because the torque induced by the lateral acceleration due to $\boldsymbol{F}_{m}$ [proportional to $\gamma \boldsymbol{a}_{c} \times \boldsymbol{p}$, see Eq. (2)] then either enhances (rising particles) or counteracts (settling) the rotation rate $\boldsymbol{\omega}$. Therefore, rotational amplitudes are suppressed for heavy particles via this mechanism. In the resonance regime, $\boldsymbol{F}_{m}$ strongly aligns with the direction of normal acceleration $N$, such that translational accelerations due to other forces also amplify the effect in this case.

Finally, to put our results into perspective, we compare them to compiled literature data in terms of $C_{d}$ vs Re in Fig. 4. The range of $C_{d}$ in the present measurements is seen to cover the full spread in the literature data with matching bounds, indicating that, at least at this level, the dynamics explored here are comparable to those encountered (nominally) without c.m. offset. The fact that, here, this variation arises from altering only the rotational dynamics is testament to the crucial importance of related parameters such as $I^{*}$ and $\gamma$. Therefore, incorporating these appears necessary for a complete description of the problem. Moreover, there is a longstanding notion [20], with mention already by Newton [8], that high levels of $C_{d}$ are associated with large path amplitudes $\hat{a} / D$. This is clearly at odds with our results at $\Gamma>1$ (but, also, with findings by others

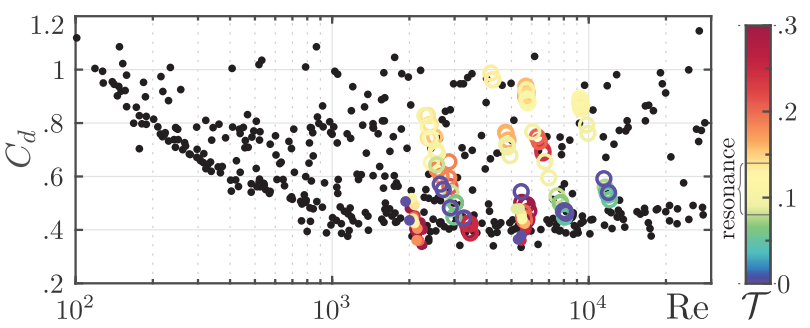

FIG. 4. Particle drag coefficients for rising and settling spheres compiled from literature (black dots) [17,19-22,25,26,57-63], and present data (color coded by $\mathcal{T}$ ) vs $\operatorname{Re}=\left\langle u_{z}\right\rangle D / \nu$. 
$[13,17,49,56])$, where $C_{d}$ remains low even though $\hat{a} / D$ is significant. Our analysis suggests that $C_{d}$ is, instead, more closely related to particle rotations.

In summary, we have provided strong evidence for how critically the overall behavior of free rising or sinking spheres in the vortex-shedding regime is related to their rotational dynamics. The revealed sensitivity to c.m. offsets as small as $\gamma=0.5 \%$ is remarkable, and therefore, this parameter is likely to play a role in many practical cases. In particular, it might affect the behavior of spheroidal bubbles [64], which are known to display spiral or zigzag motion when rising in a contaminated liquid [65-67]. In that case, a c.m. offset might arise due to the fact that surfactants are swept to the back of the bubble by the flow and we estimate (assuming $\Gamma \rightarrow 0$ and $I^{*}=1$ ) that $\gamma \approx 5 \%$ would suffice to reach a $\mathcal{T}$ value in the resonance regime. Clearly, the present findings are also useful to tailor particle behavior. In the future, it will be of particular interest to broaden the investigation to turbulent flow. Given how easily and effectively their resonance behavior can be tuned, c.m. spheres may be efficient means to "shape" turbulence by very selectively enhancing specific frequencies in the flow.

We thank Varghese Mathai, Chong Shen Ng, Chao Sun, and Detlef Lohse for insightful discussions as well as Jim Scheefhals for assisting in the experiments. This work was supported by the Netherlands Organisation for Scientific Research (NWO) under VIDI Grant No. 13477. This project has received funding from the European Research Council (ERC) under the European Union's Horizon 2020 research and innovation programme (grant agreement No. 950111 BU-PACT).

j.b.will@utwente.nl

†.j.krug@utwente.nl

[1] H. Lamb, Hydrodynamics, 6th ed. (Cambridge University Press, Cambridge, England, 1932).

[2] H. J. Lugt, Autorotation, Annu. Rev. Fluid Mech. 15, 123 (1983).

[3] P.W. Bearman, Vortex shedding from oscillating bluff bodies, Annu. Rev. Fluid Mech. 16, 195 (1984).

[4] C. H. K. Williamson and R. Govardhan, Vortex-induced vibrations, Annu. Rev. Fluid Mech. 36, 413 (2004).

[5] P. Ern, F. Risso, D. Fabre, and J. Magnaudet, Wake-induced oscillatory paths of bodies freely rising or falling in fluids, Annu. Rev. Fluid Mech. 44, 97 (2012).

[6] V. Mathai, D. Lohse, and C. Sun, Bubbly and buoyant particle-laden turbulent flows, Annu. Rev. Condens. Matter Phys. 11, 529 (2020).

[7] I. Marusic and S. Broomhall, Leonardo da Vinci and fluid mechanics, Annu. Rev. Fluid Mech. 53, 1 (2020).

[8] I. Newton, The Principia: Mathematical Principles of Natural Philosophy (University of California Press, Berkeley, 1999).

[9] J. Magnaudet and I. Eames, The motion of high-Reynoldsnumber bubbles in inhomogeneous flows, Annu. Rev. Fluid Mech. 32, 659 (2000).
[10] L. H. Zhao, H. I. Andersson, and J. J. J. Gillissen, Turbulence modulation and drag reduction by spherical particles, Phys. Fluids 22, 081702 (2010).

[11] E. Alméras, F. Risso, V. Roig, S. Cazin, C. Plais, and F. Augier, Mixing by bubble-induced turbulence, J. Fluid Mech. 776, 458 (2015).

[12] E. Alméras, V. Mathai, C. Sun, and D. Lohse, Mixing induced by a bubble swarm rising through incident turbulence, Int. J. Multiphase Flow 114, 316 (2019).

[13] F. Auguste and J. Magnaudet, Path oscillations and enhanced drag of light rising spheres, J. Fluid Mech. 841, 228 (2018).

[14] V. Mathai, X. Zhu, C. Sun, and D. Lohse, Flutter to tumble transition of buoyant spheres triggered by rotational inertia changes, Nat. Commun. 9, 1792 (2018).

[15] M. Jenny, G. Bouchet, and J. Dušek, Nonvertical ascension or fall of a free sphere in a Newtonian fluid, Phys. Fluids 15, L9 (2003).

[16] M. Horowitz and C. H. K. Williamson, Critical mass and a new periodic four-ring vortex wake mode for freely rising and falling spheres, Phys. Fluids 20, 101701 (2008).

[17] M. Jenny, J. Dušek, and G. Bouchet, Instabilities and transition of a sphere falling or ascending freely in a Newtonian fluid, J. Fluid Mech. 508, 201 (2004).

[18] C. H. J. Veldhuis, A. Biesheuvel, L. van Wijngaarden, and D. Lohse, Motion and wake structure of spherical particles, Nonlinearity 18, C1 (2005).

[19] C. H. J. Veldhuis and A. Biesheuvel, An experimental study of the regimes of motion of spheres falling or ascending freely in a Newtonian fluid, Int. J. Multiphase Flow 33, 1074 (2007).

[20] M. Horowitz and C.H.K. Williamson, The effect of Reynolds number on the dynamics and wakes of freely rising and falling spheres, J. Fluid Mech. 651, 251 (2010).

[21] A. W. Preukschat, Measurements of drag coefficients for falling and rising spheres in free motion, Master's thesis, California Institute of Technology, 1962, http://dx.doi.org/ 10.7907/94MG-ZS76.

[22] U. Shafrir, Horizontal Oscillations of Falling Spheres, Technical Report, Air Force Cambridge Research Labs, 1965.

[23] E. B. Christiansen and D. H. Barker, The effect of shape and density on the free settling of particles at high Reynolds numbers, AlChE J. 11, 145 (1965).

[24] D. G. Karamanev and L. N. Nikolov, Free rising spheres do not obey Newton's law for free settling, AIChE J. 38, 1843 (1992).

[25] D. G. Karamanev, C. Chavarie, and R. Mayer, Dynamics of the free rise of a light solid sphere in liquid, AIChE J. 42, 1789 (1996).

[26] C. H. J. Veldhuis, A. Biesheuvel, and D. Lohse, Freely rising light solid spheres, Int. J. Multiphase Flow 35, 312 (2009).

[27] K. Namkoong, J. Y. Yoo, and H. G. Choi, Numerical analysis of two-dimensional motion of a freely falling circular cylinder in an infinite fluid, J. Fluid Mech. 604, 33 (2008).

[28] V. Mathai, X. Zhu, C. Sun, and D. Lohse, Mass and Moment of Inertia Govern the Transition in the Dynamics and Wakes of Freely Rising and Falling Cylinders, Phys. Rev. Lett. 119, 054501 (2017).

[29] E. Loth, Lift of a spherical particle subject to vorticity and/or spin, AIAA J. 46, 801 (2008). 
[30] C. Cummins, M. Seale, A. Macente, D. Certini, E. Mastropaolo, I. M. Viola, and N. Nakayama, A separated vortex ring underlies the flight of the dandelion, Nature (London) 562, 414 (2018).

[31] A. Nemes, T. Dasari, J. Hong, M. Guala, and F. Coletti, Snowflakes in the atmospheric surface layer: Observation of particle-turbulence dynamics, J. Fluid Mech. 814, 592 (2017).

[32] C. Li, K. Lim, T. Berk, A. Abraham, M. Heisel, M. Guala, F. Coletti, and J. Hong, Settling and clustering of snow particles in atmospheric turbulence, J. Fluid Mech. 912, A49 (2021).

[33] T. Zeugin, Q. Krol, I. Fouxon, and M. Holzner, Sedimentation of snow particles in still air in stokes regime, Geophys. Res. Lett. 47, e2020GL087832 (2020).

[34] M. W. McCorquodale and C. Westbrook, Trail: A novel approach for studying the aerodynamics of ice particles, Q. J. R. Meteorol. Soc. 147, 589 (2021).

[35] M. W. McCorquodale and C. Westbrook, Trail part 2: A comprehensive assessment of ice particle fall speed parametrisations, Q. J. R. Meteorol. Soc. 147, 605 (2021).

[36] J. F. Richardson and W. N. Zaki, The sedimentation of a suspension of uniform spheres under conditions of viscous flow, Chem. Eng. Sci. 3, 65 (1954).

[37] E. Meiburg and B. Kneller, Turbidity currents and their deposits, Annu. Rev. Fluid Mech. 42, 135 (2010).

[38] R. Sowmeyan and G. Swaminathan, Evaluation of inverse anaerobic fluidized bed reactor for treating high strength organic wastewater, Bioresource technology : biomass, bioenergy, biowastes, conversion technologies, biotransformations, production technologies 99, 3877 (2008).

[39] L. K. Clark, M. H. DiBenedetto, N. T. Ouellette, and J. R. Koseff, Settling of inertial nonspherical particles in wavy flow, Phys. Rev. Fluids 5, 124301 (2020).

[40] M. Tanaka, K. Tajiri, H. Nishida, and M. Yamakawa, Effect of eccentric mass distribution on the motion of spherical particles in shear flows, J. Fluids Eng. 142, 031105 (2020).

[41] S. Yasseri, Experiment of free-falling cylinders in water, Underwater Technol. 32, 177 (2014).

[42] B. R. Angle, M. J. Rau, and M. L. Byron, Effect of mass distribution on falling cylindrical particles at intermediate Reynolds numbers, in ASME 2019 Fluids Engineering Division Summer Meeting (American Society of Mechanical Engineers, New York, 2019), https://doi.org/10.1115/AJK Fluids2019-5458.

[43] A. Roy, R. J. Hamati, L. Tierney, D. L. Koch, and G. A. Voth, Inertial torques and a symmetry breaking orientational transition in the sedimentation of slender fibres, J. Fluid Mech. 875, 576 (2019).

[44] G. Mougin and J. Magnaudet, The generalized Kirchhoff equations and their application to the interaction between a rigid body and an arbitrary time-dependent viscous flow, Int. J. Multiphase Flow 28, 1837 (2002).

[45] D. Fabre, F. Auguste, and J. Magnaudet, Bifurcations and symmetry breaking in the wake of axisymmetric bodies, Phys. Fluids 20, 051702 (2008).

[46] C. H. K. Williamson and R. Govardhan, Vortex-induced vibrations, Annu. Rev. Fluid Mech. 36, 413 (2004).

[47] A. Belmonte, H. Eisenberg, and E. Moses, From Flutter to Tumble: Inertial Drag and Froude Similarity in Falling Paper, Phys. Rev. Lett. 81, 345 (1998).
[48] V. Mathai, M. W. M. Neut, E. P. van der Poel, and C. Sun, Translational and rotational dynamics of a large buoyant sphere in turbulence, Exp. Fluids 57, 51 (2016).

[49] J. B. Will, V. Mathai, S. G. Huisman, D. Lohse, C. Sun, and D. Krug, Kinematics and dynamics of freely rising spheroids at high Reynolds numbers, J. Fluid Mech. 912, A16 (2021).

[50] See Supplemental Material at http://link.aps.org/ supplemental/10.1103/PhysRevLett.126.174502 for details on the experimental setup and procedures. The supplement further contains tabulated experimental parameters and results as well as a sample video of particle dynamics.

[51] R. E. D. Bishop and A. Y. Hassan, The lift and drag forces on a circular cylinder oscillating in a flowing fluid, Proc. R. Soc. Ser. A 277, 51 (1964).

[52] P. W. Bearman and E. D. Obasaju, An experimental study of pressure fluctuations on fixed and oscillating square-section cylinders, J. Fluid Mech. 119, 297 (1982).

[53] S. K. Jordan and J. E. Fromm, Oscillatory drag, lift, and torque on a circular cylinder in a uniform flow, Phys. Fluids 15, 371 (1972).

[54] G. Bouchet, M. Mebarek, and J. Dušek, Hydrodynamic forces acting on a rigid fixed sphere in early transitional regimes, Eur. J. Mech. B 25, 321 (2006).

[55] R. Zimmermann, Y. Gasteuil, M. Bourgoin, R. Volk, A. Pumir, and J.-F. Pinton, Rotational Intermittency and Turbulence Induced Lift Experienced by Large Particles in a Turbulent Flow, Phys. Rev. Lett. 106, 154501 (2011).

[56] W. Zhou and J. Dušek, Chaotic states and order in the chaos of the paths of freely falling and ascending spheres, Int. J. Multiphase Flow 75, 205 (2015).

[57] P. B. MacCready and H. R. Jex, Study of sphere motion and balloon wind sensors, Technical Report No. NASA TM X53089, 1964.

[58] G. Kuwabara, S. Chiba, and K. Kono, Anomalous motion of a sphere falling through water, J. Phys. Soc. Jpn. 52, 3373 (1983).

[59] G. E. Stringham, D. B. Simons, and H. P. Guy, The behavior of large particles falling in quiescent liquids, Professional Paper, U.S. Geological Survey 562-C, 1969, https://doi.org/ 10.3133/pp562C.

[60] H. S. Allen, The motion of a sphere in a viscous fluid: III, Philos. Mag. 50, 519 (1900).

[61] H. Liebster, Über den widerstand von kugeln, Ann. Phys. (Leipzig) 387, 541 (1927).

[62] R. G. Lunnon, Fluid resistance to moving spheres, Proc. R. Soc. A 118, 680 (1928).

[63] J. L. Boillat and W. H. Graf, Settling velocity of spherical particles in calm water, J. Hydraul. Div. Am. Soc. Civ. Eng. 107, 1123 (1981).

[64] G. Mougin and J. Magnaudet, Path Instability of a Rising Bubble, Phys. Rev. Lett. 88, 014502 (2001).

[65] W. L. Haberman and R. K. Morton, An experimental investigation of the drag and shape of air bubbles rising in various liquids, Technical Report, David Taylor Model Basin Report No 802, 1953.

[66] P. G. Saffman, On the rise of small air bubbles in water, J. Fluid Mech. 1, 249 (1956).

[67] R. A. Hartunian and W. R. Sears, On the instability of small gas bubbles moving uniformly in various liquids, J. Fluid Mech. 3, 27 (1957). 